
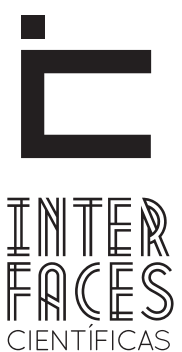

SAÚDE E AMBIENTE

ISSN IMPRESSO 2316-3313

E - ISSN 2316-3798

DOI - 10.17564/2316-3798.2018v7n1p9-16

\title{
AUTONOMIA DAS PESSOAS COM DEFICIÊNCIA VISUAL EM INSTTTUIC̣̃̃ES DE ENSINO SUPERIOR
}

\author{
AUTONOMY OF PERSONS WITH VISUAL DEFICIENCY IN INSTITUTIONS OF HIGHER EDUCATION
}

AUTONOMÍA DE LAS PERSONAS CON DISCAPACIDAD VISUAL EN INSTITUCIONES DE ENSEÑANZA SUPERIOR

Luana Bernardi ${ }^{1}$

Josivaldo Godoy da Silva

Valter Aragão do Nascimento ${ }^{5}$
Ana Carla Gomes Rosa²

Andreia Conceição Milan Brochado Antoniolli ${ }^{4}$

\section{RESUMO}

A deficiência visual é a deficiência mais prevalente no Brasil. Embora haja um incentivo por parte de políticas governamentais para que se amplie o acesso à educação, sabe-se que a adaptação de ambientes a estes indivíduos ainda é precária. Assim, o objetivo deste artigo é discorrer sobre a importância das pessoas com deficiência visual no ensino superior e as diferentes ferramentas que podem ser implantadas nestas instituições com o objetivo de ampliar a acessibilidade a estes locais. Estes indivíduos necessitam de um ensino superior adaptado para que se possibilite maior inclusão e aumento da autonomia. Destaca-se o uso de dispositivos móveis, a presença de pisos táteis, materiais em formato eletrônico, o preparo dos educadores para trabalhar com este público, bem como o treinamento prévio do deficiente visual para fazer uso destas ferramentas.

\section{PALAVRAS-CHAVE}

Cegueira. Educação Superior. Tecnologia. Equipamentos de Autoajuda. Tecnologia da Informação. 


\section{ABSTRACT}

Visual deficiency is the most prevalent deficiency in Brazil. Although there is an incentive by government policies to increase access to education, it is known that the adaptation of environments to these individuals is still precarious. Thus, the objective of this article is to discuss the importance of people with visual impairment in higher education and the different tools that can be implemented in these institutions in order to increase accessibility to these places. These individuals need a higher education adapted to allow greater inclusion and increase au- tonomy. Of particular note are the use of mobile devices, the presence of tactile floors, electronic materials, the preparation of educators to work with this public, as well as the prior training of the visually impaired to make use of these tools.

\section{KEYWORDS}

Blindness. Higher education. Technology. Self-Help Devices. Information Technology.

\section{RESUMEN}

La deficiencia visual es la deficiencia más prevalente en Brasil. Aunque hay un incentivo por parte de políticas gubernamentales para que se amplíe el acceso a la educación, se sabe que la adaptación de ambientes a estos individuos todavía es precaria. Así, el objetivo de este artículo es discurrir sobre la importancia de las personas con discapacidad visual en la enseñanza superior y las diferentes herramientas que pueden ser implantadas en estas instituciones con el objetivo de ampliar la accesibilidad a estos locales. Se optó por artículo de reflexión en las que se utilizaron las bases de datos como Scielo y Lilacs. Estos individuos necesitan una enseñanza su- perior adaptada para que se posibilite mayor inclusión y aumento de la autonomía. Se destaca el uso de dispositivos móviles, la presencia de pisos táctiles, materiales en formato electrónico, la preparación de los educadores para trabajar con este público, así como el entrenamiento previo del deficiente visual para hacer uso de estas herramientas.

\section{PALABRAS CLAVE}

Ceguera. Educación universitaria. Tecnología. Equipos de autoayuda. Tecnología de la información 


\section{INTRODUÇÃO}

Segundo Estatuto da Pessoa com Deficiência (2015) considera-se pessoa com deficiência aquelas que têm impedimento de longo prazo de natureza física, mental, intelectual ou sensorial, os quais, em interação com diversas barreiras, podem obstruir sua participação plena e efetiva na sociedade em igualdade de condições com as demais pessoas (BRASIL, 2015). Entre os diversos tipos de deficiências que podem acometer um indivíduo, tem-se a deficiência visual ou cegueira e a baixa visão. Dentro deste campo de definições, em 2002, durante o Congresso Internacional de Oftalmologia realizado em Sydney, Austrália, a cegueira passou a ser compreendida como a perda total ou quase total da visão, na qual os indivíduos necessitam de recursos que substituam sua capacidade de enxergar. Na baixa visão, o sujeito apresenta resquícios da visão, necessitando de recursos ópticos que a estimulem.

Segundo o Relatório Mundial sobre Deficiência (2011) estima-se que cerca de 39 milhões de pessoas no mundo são cegas e outras 246 milhões tem baixa visão (OMS, 2011). Além disso, acredita-se que os países em desenvolvimento são os mais acometidos, com $80 \%$ da cegueira mundial (CONGDON et al., 2003). A nível nacional estudos vêm sendo desenvolvidos com o intuito de alertar o aumento do número de casos. Em recente inquérito populacional, observou-se por meio de dados provenientes da Pesquisa Nacional de Saúde, que a deficiência visual é o tipo de deficiência mais prevalente no Brasil (3,6\%), sua ocorrência tende a aumentar conforme a idade, sendo as faixas de 40 a 59 anos $(5,1 \%)$ e 60 anos ou mais $(11,5 \%)$ as mais acometidas e, se destaca principalmente na região Sul $(5,9 \%)$, entre indivíduos que residem no meio rural (4,7\%) (MALTA et al., 2016).

Salomão e outros autores (2009) analisando os dados de dois importantes estudos populacionais sobre a ocorrência de deficiência visual em escolares e adultos, alertaram ser um problema significativo em brasileiros mais velhos, reforçando a necessidade de implementação de programas de prevenção de cegueira para pessoas idosas com ênfase nos indivíduos sem escolaridade.
O impacto socioeconômico e o número crescente de pessoas acometidas por esta deficiência, tornam-na um importante problema de saúde pública (CONGDON et al., 2003). Malta e outros autores (2016) justificam que a alta prevalência da deficiência visual no país pode ter relação com a sua tipificação, segundo a legislação nacional. Isso porque já é considerado deficiente visual o indivíduo que tem a presença de baixa visão em um ou ambos os olhos (BRASIL, 2004). Em vista destas informações, dois fatores passam a ser avaliados: o primeiro diz respeito ao uso de serviços de reabilitação por estas pessoas; e o segundo à disponibilidade em locais púbicos e privados de tecnologias assistivas que contribuam com a inserção do deficiente visual na sociedade.

Indivíduos acometidos por deficiência visual são reconhecidos como aqueles que menos frequentam serviços de reabilitação no Brasil, comparados a outros tipos de deficiências (MALTA et al., 2016). No entanto, embora haja um incentivo por parte de políticas governamentais para que se amplie o acesso à educação, inclusão social, acessibilidade e atenção à saúde destas pessoas (BRASIL, 2008), sabe-se que a adaptação de ambientes aos deficientes visuais ainda é precária e escassa. Isso remete a importância da autonomia da pessoa com deficiência visual, que pode ser compreendida como a "própria autoridade" para guiar sua vida pessoal e social (ACIEM; MAZZOTTA, 2013).

0 referido trabalho trata-se de um artigo de reflexão, embasados em legislação e estudos brasileiros e do exterior, que se propunham a "discorrer sobre a importância da inclusão das pessoas com deficiência visual em instituições de ensino superior", "dissertar sobre problemas relacionados com a acessibilidade das pessoas com deficiência visual em instituições de ensino superior" e "apresentar estratégias para aumentar a acessibilidade das pessoas com deficiência visual em instituições de ensino superior".

Essa reflexão é amadurecimento de trabalho no âmbito de Programa de Pós-graduação Stricto Sensu na área de Saúde e visa refletir sobre a importância das pessoas com deficiência visual no ensino superior e as diferentes ferramentas que podem ser implan- 
tadas nestas instituições com o intuito de ampliar a acessibilidade destes indivíduos a estes locais.

\section{A INCLUSÃO DAS PESSOAS COM DEFICIÊNCIA VISUAL EM INSTITUIÇÕES DE ENSINO SUPERIOR}

O termo 'inclusão social' pode ser compreendido como a participação de um indivíduo em um meio social, no qual ele tem os mesmos direitos e deveres que os demais (FIGUEIREDO et al., 2011). Partindo dessa premissa, a inclusão das pessoas com deficiência, constitui-se em um movimento que busca garantir a estas pessoas, entre outros direitos, 0 acesso à educação e a facilitação da sua mobilidade (COSTA, 2016). Segundo Coulon (2008) para que um recém-chegado em uma universidade se torne um estudante, ele deve passar por três etapas sucessivas: "o tempo do estranhamento, o tempo do aprendizado e o tempo da afiliação". No entanto, um estudante como o deficiente visual necessita de um suporte especial ofertado pela instituição para que ele consiga percorrer os três tempos citados.

Figueiredo e outros autores (2011) atestam que o número de estudantes com deficiência na educação superior ainda é escasso. Alguns fatores podem estar relacionados a este fato, sendo que um deles pode se referir às dificuldades de acessibilidade encontradas nas instituições que, muitas vezes, impedem o acesso adequado aos diferentes espaços de uma universidade. Quanto a isso, a Lei no 10.098 , de 19 de dezembro de 2004, que traz normas para a supressão de barreiras e de obstáculos nas vias e espaços públicos, no mobiliário urbano, na construção e reforma de edifícios e nos meios de transporte e comunicação, promovendo a acessibilidade das pessoas com deficiência ou com mobilidade reduzida, a qual estabelece que:

Os estabelecimentos de ensino de qualquer nível, etapa ou modalidade, públicos ou privados, proporcionarão condições de acesso e utilização de todos os seus ambientes ou compartimentos para pessoas portadoras de deficiência ou com mobilidade reduzida, inclusive salas de aula, bibliotecas, auditórios, ginásios e instalações desportivas, laboratórios, áreas de lazer e sanitários.

A exigência exposta acima, quando direcionada para a realidade de muitas instituições brasileiras, apenas demonstra que o direito a acessibilidade das pessoas cegas está comprometido. No entanto, Dallabrida e Lunardi (2008) explicam que o número de estudantes deficientes visuais que alcançam as Instituições de Ensino Superior (IES) está aumentando, devido ao maior uso do sistema Braille durante a sua escolarização. Isso gera uma preocupação, uma vez que estas instituições não estão se adequando à chegada destes deficientes, não lhes proporcionando um ambiente de inclusão.

Assim, além de ter o conhecimento das dificuldades que existem para a inclusão das pessoas com deficiência visual como cidadãos, é essencial conhecer as possibilidades existentes para que consigam vencer os obstáculos colocados por esta deficiência como, por exemplo, a realização do vestibular até o momento da aprovação bem como, a carência de bibliografias impressas em alfabeto Braille, o que os torna dependente dos colegas para leitura, necessitando da reprodução de trabalhos e provas, sendo que, em muitos casos, estes serviços são cobrados (BRUMER et al., 2004).

Diante das necessidades aumentadas do grupo de indivíduos com deficiência visual em IES, é de extrema importância que esta deficiência seja minimizada nestes espaços e, que ao mesmo tempo, as habilidades deste público possam ser desenvolvidas de forma adequada. Isso pode ser alcançado por meio de adaptações tanto nos materiais utilizados quanto nos métodos de ensino, permitindo que o deficiente visual tenha a mesma oportunidade de se destacar profissionalmente como outra pessoa que não tem a visão prejudicada. Assim, este grupo de indivíduos necessita de um ensino superior adaptado que leve em consideração suas limitações no âmbito da aprendizagem, interação social, locomoção e operação geral (ANDREW, 2015).

Para que se possibilite maior inclusão destas pessoas e aumento da sua autonomia, devem-se criar 
situações dentro de instituições acadêmicas que permitam aos deficientes lidar com as atividades de ensino que ocorrem nestes locais, o que pode ser alcançado do fornecimento de materiais específicos e de uma educação mais qualitativa. Esta oportunidade de acesso ao ensino superior com qualidade, impacta no maior conhecimento dos deficientes visuais, de seus deveres, além de permitir que eles consigam cuidar melhor de si próprios e das pessoas ao seu redor, como a família (ANDREW, 2015). Citam-se em seguida algumas formas de ampliar a inserção igualitária dos deficientes visuais em instituições de ensino, por meio de estratégias que podem proporcionar comodidade e praticidade nos locais visitados.

\section{INTRODUÇÃO DE ESTRATÉGIAS DE INCLUSÃO PARA PESSOAS COM DEFICIÊNCIA VISUAL EM INSTITUIÇÕES DE ENSINO SUPERIOR}

A inclusão das pessoas com deficiência visual em IES esbarra em alguns problemas que inibem a presença destes indivíduos em salas de aula. Andrew (2015) destaca que as ofertas de oportunidades de educação para estas pessoas muitas vezes são contentáveis, devido aos problemas arquitetônicos nas instituições, à atitude negativa de alguns indivíduos dentro das instituições, à inadequação dos materiais/equipamentos fornecidos, bem como ao elevado custo tanto dos materiais quanto da educação para este grupo de indivíduos. Brumer e outros autores (2004) ressaltam problemas como o treinamento e/ou preparo deficitário dos professores para trabalhar com este público, a falta de financiamento adequado das instituições e a falta de legislações e rigidez administrativa eficientes que se preocupem com a inclusão dos deficientes visuais.

Pressupõe-se, considerando que o desenvolvimento nacional está inter-relacionado com a educação, que os problemas aqui citados devam ser discutidos e solucionados. Assim, é necessário um ensino superior que reduza as incapacidades dos deficientes visuais e que propicie o desenvolvimento das habilidades destes indivíduos (ANDREW, 2015). Em estudo de Foley e Masingyla (2015) no Quênia, explorou-se o uso de dispositivos móveis como tecnologia assistiva para alunos com deficiência visual em ambientes educacionais com recursos limitados e, verificaram que a inserção da tecnologia móvel possibilitou aos alunos ampliação do acesso à educação e dos meios de participar da vida cotidiana, além de aumentar sua prática na comunidade.

Embora uma pequena fração de alunos com deficiência visual sejam percebidos na educação superior, notam-se também dificuldades relacionadas ao relacionamento professor-aluno com deficiência visual. Assim, ainda são grandes as dificuldades encontradas no processo de inclusão, tanto devido a lacunas na formação docente quanto devido à postura adotada pelo professor em sala de aula.

Há a ausência na grade curricular dos professores de conteúdos que tratem sobre deficiência visual e que desenvolvam habilidades destes para trabalhar com alunos que apresentam deficiência. Esta carência no processo de ensino dos professores provoca o desconhecimento, muitas vezes, até do conceito de deficiência visual. Por isso, sugere-se a implantação de disciplinas referentes à educação inclusiva na grade curricular dos graduandos em licenciatura e o investimento em formação continuada (REIS et al., 2010).

Entre as estratégias que podem auxiliar pessoas com deficiência visual a circular e serem guiados com segurança dentro das IES, existem os "indicadores de superfície tátil do solo", como os pisos táteis. A pessoa pode fazer uso destes indicadores, colocando ambos os pés sobre o piso, ou não pisando nos indicadores, mas tocando-lhes com bastões longos ou, ainda, pisando com somente um pé (REIS et al., 2010). De qualquer forma, eles são úteis para guiar o trajeto que está sendo percorrido pelo indivíduo cego.

Nandini e Seeja (2017) no seu trabalho propõem um sistema operacional sonoro, que pode ser incorporado a uma bengala usada pelos deficientes visuais no qual é previamente informado ao usuário, qual a 
melhor rota seguir, disponibilizando a distância que irá percorrer, prevenindo erros de trajetos.

Em trabalho recente de Urbas e outros autores (2016), suscita-se o problema relacionado ao material utilizado para construção do piso tátil. Os autores ressaltam que ele pode ser feito de diferentes materiais como madeira, metal, plástico, bem como por diferentes técnicas que requerem muita engenhosidade. Por exemplo, o piso tátil pode ser feito de papel microemcapsulado, no entanto, sua resistência e durabilidade tendem a ser limitadas, o que também é refletido no seu tempo de uso.

Com base nesta prerrogativa, é proposta a produção de planos táteis, usando a impressão 3D, a qual também pode ser utilizada para impressão de outras ferramentas para cegos, como placas em alfabeto Braille e o mapa em 3D. Embora os custos com a impressão a partir desta nova técnica ainda sejam elevados, reitera-se que é mais resistente e durável, sendo uma alternativa interessante na área de impressão para pessoas com deficiência visual.

No que concerne às dificuldades de acessibilidade das pessoas com deficiência visual nas IES, esta fica visível nos campos de estágio propostos por estas instituições, os quais não estão preparados para oferecer a estes indivíduos o suporte necessário para a sua qualificação profissional.

Segundo Selau e outros autores (2017), não são apenas obstáculos atitudinais e arquitetônicos que o deficiente visual enfrenta no âmbito da educação superior, mas enfrentam a falta de recursos específicos para aprendizagem, faz-se necessário novas estratégias metodológicas como por exemplo a aquisição de instrumental tecnológico e material adaptado.

No estudo de Johnston e outros autores (2016), foi verificado que o ambiente hospitalar apresenta limitações para o preparo clínico das pessoas com deficiência visual que estão sendo educados para se tornar fisioterapeuta. O fator que contribuiu com esta formação profissional, a partir da intervenção proposta, foi a inclusão de um suporte tecnológico para que o aluno pudesse ter acesso aos manuais que até então só estavam disponíveis em formato eletrônico. Assim, os materiais passaram a estar acessíveis também por meio de gravações de áudio ou on-line, para que um formato ampliado de tela pudesse ser usado.

Entende-se que o apoio às pessoas com deficiência visual a partir de várias estratégias de inclusão é um processo essencial para a permanência destes indivíduos no ensino superior. Alves e outros autores (2009) verificaram a aplicação de tecnologia assistiva, principalmente de tecnologia da informação, na educação de alunos cegos e de baixa visão, a partir das percepções de professores de alguns municípios de São Paulo. Neste trabalho, observaram que os usos de programas específicos de computador são importantes por melhorar as habilidades de leitura e escrita, bem como a comunicação com o mundo em igualdade de condições, melhorando assim a qualidade de vida e facilitando o processo de aprendizagem. No entanto, a falta de infraestrutura e apoio pedagógico limita muitas vezes o uso da tecnologia de informação no ambiente educacional.

\section{CONSIDERAÇÕES FINAIS}

0 acesso e permanência das pessoas com deficiência visual em IES está relacionado com o processo de aceitação destes indivíduos nos mais diversos setores da sociedade, uma vez que com o estímulo à educação continuada, as pessoas cegas conseguem alcançar espaço no mercado de trabalho e, assim, passam a ser vistas também como contribuintes para o desenvolvimento nacional. Entretanto, nota-se uma escassez de literatura referente aos problemas enfrentados por este público e nestas instituições, o que pode evidenciar a falta de interesse por parte de profissionais e pesquisadores nesta área. Acredita-se que mais projetos devam ser elaborados e colocados em prática, para facilitar o acesso das pessoas com deficiência visual ao ensino superior.

A presença de estratégias para a inclusão das pessoas com deficiência visual nestas instituições, como o uso de tecnologias assistivas, incluindo o preparo dos professores para trabalhar com este público em sala de aula e nos campos de atuação, ainda perma- 
necem escassas. No entanto, percebe-se um interesse por parte de estudiosos em discutir e expor sobre diferentes métodos de inclusão e aprendizagem das pessoas com deficiência visual. Logo, as possibilidades levantadas nesta revisão poderão servir como base para serem implantadas em outras instituições de ensino e também para fomentar a importância do desenvolvimento de projetos de inclusão para indivíduos cegos no meio acadêmico.

\section{REFERÊNCIAS}

ACIEM, T.M.; MAZZOTTA, M.J.S. Autonomia pessoal e social de pessoas com deficiência visual após reabilitação. Rev Bras Oftalmol., v.72, n.4, p.261267, 2013.

ALVES, C.C.F. et al. Assistive technology applied to education of students with visual impairment. Rev Panam Salud Públ., v.26, n.2, p.148-152, 2009.

ANDREW, O.A. The challenges of educating the visually impaired and quality assurance in tertiary institutions of learning in Nigeria. Int J Educ Admin Pol Stud., v.7, n.7, p.129-133, 2015.

BRASIL. Presidência da República. Decreto n 5.296, de 2 de dezembro de 2004. Regulamenta as Leis nos 10.048 , de 8 de novembro de 2000, que dá prioridade de atendimento às pessoas que especifica, e 10.098, de 19 de dezembro de 2000, que estabelece normas gerais e critérios básicos para a promoção da acessibilidade das pessoas portadoras de deficiência ou com mobilidade reduzida, e dá outras providências. Disponível em: <http://www.planalto. gov.br/ccivil_03/_Ato2004-2006/2004/Decreto/ D5296.htm> Acesso em: 26 jul. 2018

BRASIL. Ministério da Saúde (MS). Política Nacional de Saúde da Pessoa Portadora de Deficiência. Brasília: MS, 2008
BRASIL. Presidência da República. Lei 13.146, de 6 de julho de 2015. Institui a Lei Brasileira de Inclusão da Pessoa com Deficiência (Estatuto da Pessoa com Deficiência). Diário Oficial da União, 7 jul. 2015.

BRUMER, A.; PAVEI, K.M.D.G. Saindo da "escuridão": perspectivas da inclusão social, econômica, cultural e política dos portadores de deficiência visual em Porto Alegre. Sociologias, n.11, p.300-327, 2004.

BELAY, T.E. African perspective on visual impairments ICT's and policies: a personal experience, a variety of perspectives and technological solution. In: UNESCO at the World Summit on the Information Society Workshop on ICT and persons with disabilities Tunis, Tunisia. 2005.

CONGDON, N.G. et al. Important causes of visual impairment in the world today. J Am Med Assoc., v.290, p.2057-2060, 2003.

COULON, A. A condição de estudante: a entrada na vida universitária. Salvador: EDUFBA, 2008.

DALLABRIDA, A.M.; LUNARDI, G.M. 0 acesso negado e a reiteração da dependência: a biblioteca e o seu papel no processo formativo de indivíduos cegos. Cad Centro Est Educ Soc., v.28, n.75, p.191-208, 2008.

FOLEY, A.R.; MASINGILA, J.O. The use of mobile devices as assistive technology in resource-limited environments: access for learners with visual impairments in Kenya. J Disabil Rehabil Assist Techn., v.10, n.4, p.332-339, 2015.

FIGUEIREDO, A.C. et al. Acessibilidade e vida universitária: pontuações sobre a educação inclusiva. In: SAMPAIO, S.M.R. (Org.). Observatório da vida estudantil: primeiros estudos [on-line], Salvador: EDUFBA, 2011. p.187-207.

JOHNSTON, K.N. et al. Reconsidering inherent requirements: a contribution to the debate from the 
clinical placement experience of a physiotherapy student with vision impairment. BMC Med Educ., v.16, p.74, 2016.

MALTA, D.C. et al. Prevalência autorreferida de deficiência no Brasil, segundo a Pesquisa Nacional de Saúde, 2013. Ciênc Saúde Col., v.21, n.10, p.3253-3264, 2016.

NANDINI, D.; SEEJA, K.R. A novel path-planning algorithm for visually impaired people. JKSUCIS 2017. Disponível em: <https://doi.org/10.1016/j. jksuci.2017.03.005>. Acesso em: 23 jul. 2018.

REIS, M.X. et al. A formação do professor para o ensino superior: prática docente com alunos com deficiência visual. Educ Rev., v.26, n.1, p.111-130, 2010.
SALOMAO, S.R. et al. Visual impairment and blindness: an overview of prevalence and causes in Brazil. An Acad Bras Ciên., v.81, n.3, p.539-549, 2009.

SELAU, B. et al. Estudantes cegos na educação superior: o que fazer com os possíveis obstáculos? Acta Sci Educ., v.39, n.4, p.431-440, 2017.

URBAS, R. et al. Development of tactile floor plan for the blind and the visually impaired by 3D printing technique. J Graph Eng Des., v.7, n.1, 2016

OMS. Organização Mundial da Saúde. Relatório mundial sobre a deficiência 2011. Disponível em: <http://www.pessoacomdeficiencia.sp.gov. br/usr/share/documents/RELATORIO_MUNDIAL_ COMPLETO.pdf>. Acesso em: 26 jul. 2018.

E-mail: aragao60@hotmail.com 